\title{
Radiation and Differential Diagnosis of Computed Tomography (CT) Data for Coronavirus Infection "COVID-19" with Clinical Examples
}

\author{
Uulzhan Shermatova1, Elnura Boronbaeva2, Meerim Murzaibragimova3, \\ Kaldykul Dushimbekova4, Fazliddin Muidinov5, Kyialbek Sakibaev6, \\ Bektursun Avazbekov ${ }^{1}$, Zhypargul Abdullaeva ${ }^{6,7^{*}}$ (1)
}

\footnotetext{
${ }^{1}$ Department of Infectious Diseases, Medical Faculty, Osh State University, Osh, Kyrgyzstan

${ }^{2}$ Department of Propaedeutic Pediatrics, Kyrgyz State Medical Academy Named after Akhunbaev, Bishkek, Kyrgyzstan ${ }^{3}$ Department of Family Medicine, Kyrgyz State Medical Institute Named after Danyarov, Bishkek, Kyrgyzstan

${ }^{4}$ Department of Phthisiopulmonology, Kyrgyz State Medical Institute Named after Danyarov, Bishkek, Kyrgyzstan

${ }^{5}$ Department of Public Health, Medical Faculty, Osh State University, Osh, Kyrgyzstan

${ }^{6}$ Department of Anatomy, Histology and Normal Physiology, International Medical Faculty, Osh State University, Osh, Kyrgyzstan

${ }^{7}$ Science and Research Department, Osh State University, Osh, Kyrgyzstan

Email: *jypar.science@oshsu.kg
}

How to cite this paper: Shermatova, U. Boronbaeva, E., Murzaibragimova, M., Dushimbekova, K., Muidinov, F., Sakibaev, K., Avazbekov, B. and Abdullaeva, Z. (2021) Radiation and Differential Diagnosis of Computed Tomography (CT) Data for Coronavirus Infection "COVID-19" with Clinical Examples. Advances in Infectious Diseases, 11, 366-374.

https://doi.org/10.4236/aid.2021.114033

Received: October 4, 2021

Accepted: November 12, 2021

Published: November 15, 2021

Copyright $\odot 2021$ by author(s) and Scientific Research Publishing Inc. This work is licensed under the Creative Commons Attribution International License (CC BY 4.0).

http://creativecommons.org/licenses/by/4.0/

\begin{abstract}
This article is presenting data from a retrospective analysis of medical records and computed tomography (CT) scans of patients' chests with coronavirus infection "COVID-19" who applied to the diagnostic center of URFA in Osh during the first wave of the pandemic in the Kyrgyz Republic, with a description of individual clinical cases and their differential diagnosis. Chest computed tomography is one of the main methods in visual diagnosis of pneumonia in COVID-19 in hospitalized patients, which allows determining signs, symptoms for effective treatment.
\end{abstract}

\section{Keywords}

COVID-19, Coronavirus, Pneumonia, Computed Tomography, Radiation and Differential Diagnosis of Pneumonia in COVID-19, Ground Glass Opacity, Clinical Cases, Brain Damage

\section{Introduction}

The issue of differential diagnosis of patients with COVID-19 is one of relevant 
problem nowadays. There is little information in the literature available to us. Timely execution and competent interpretation of CT data allow dividing patient flows to different departments of medical institutions and providing appropriate medical care. Chest computed tomography is one of the main methods in visual diagnosis of pneumonia in COVID-19 in hospitalized patients, along with PCR diagnostics and serological studies (ELISA Ig G and M), which makes this study most relevant. The polymerase chain reaction (PCR) test is very specific but has a lower sensitivity of $65 \%$ - 95\%, which means that test can be negative even if a patient is infected. Another problem is waiting time for test results and laboratory data (CRP, D-dimers, procalcitonin, CANCER), which can take more than 24 hours.

Very common radiological findings in patients with COVID-19 were found as ground-glass opacity in their lungs, cardiovascular system, gastrointestinal tract and in the kidney [1] [2] [3].

According to A.S. Vinokurov et al., CT studies in 100\% lung lesions were manifested by areas of compaction of the "ground glass" type, in $61.9 \%$ combined with reticular striation due to intralobular interstitium, in $33.3 \%$ with compaction consolidations. In almost half of patients (52.3\%), the lesions were localized patchwork both subpleural and in the nuclear regions, in the rest, it was localized mainly in the subpleural areas. Changes in the form of subpleural stripes, compaction lines of disc-shaped atelectasis were noted in 19\%, bilateral small pleural effusion was also present in $19 \%$, and a combined increase in intrathoracic lymph nodes (IHLN) was observed only in 1 (4.7\%) patient. No foci, cavities, massive consolidations were found in patients with coronavirus infection [4]. Under the "mask" of pneumonia of viral etiology, patients were hospitalized in hospitals whose main diseases were represented by lung cancer, metastatic lesions, tuberculosis, HIV associated pathology, bronchiectasis, pulmonary infarctions, sarcoidosis, radiation pneumonitis [5] [6], as well as more rare lungs diseases (resulting in pneumonia, nonspecific interstitial pneumonia). That is why, already at the stage of the admission department, high-quality differential diagnostics are required [7].

Multislice computed tomography (MSCT) is the most informative tool in radiological examinations for patients who are suspected to viral lung disease and allows detecting early manifestations. The severity of signs in lung damage on MSCT correlates with the severity of disease, in this regard, computed tomography is used both to diagnose the lesion and to assess the process dynamics. The diagnosis of "community-acquired pneumonia of presumably coronavirus etiology" in the context of the COVID-19 epidemic is established if the patient has confirmed lung tissue infiltration (frosted glass) on CT, epidemiological data, changes in the general clinical blood test (leukopenia, lymphopenia, increased $\mathrm{CRP}$ ) and fever is sufficient for acceptance of clinical decisions before a laboratory test for the presence of a corona viral infection, even with a negative PCR diagnosis. According to some authors, the possibility of myocarditis development [8], as well as necrotizing hemorrhagic encephalopathy with the predomi- 
nant involvement of thalamic zones [9] described.

The research purpose in this work is to study features of X-ray manifestations of pneumonia in case of coronavirus infection "COVID-19" obtained on CT and to carry out their differential diagnosis with other cases of "ground glass" symptoms. CT helps to monitor the course of disease and severity of lung involvement with the most frequent finding as bilateral ground-glass opacities [10]. A negative chest CT scan does not eliminate a diagnosis of COVID-19 infection, particularly in the early stages of the disease [11].

\section{Research Methods and Materials}

A retrospective analysis of medical records and computed tomography of patients' chest who applied for CT diagnostics at the YURFA diagnostic center in Osh during the first wave of pandemic infection was carried out, with a description of individual clinical cases for differential diagnosis. Patients were chosen according to CT scan results showing chests with coronavirus infection "COVID-19". Each patient admitted to diagnostic center with suspected infection of COVID-19 was scanned with 16-slice scanner, X-ray tube 5.0 MTU, a minimum scanning time $0.8 \mathrm{~s}$, maximum field of view $500 \mathrm{~mm}$. The operating parameters of the X-ray tube tomograph were: voltage $100 \mathrm{~mA}$, currency $120 \mathrm{kV}$, time of one tube revolution $0.6 \mathrm{sec}$. The thickness of slices in images was observed as $5 \mathrm{~mm}$. In addition, according to results of reconstruction $(1.025 \mathrm{~mm})$, two series of images with different stiffness factors were obtained in pulmonary and mediastinal windows. We carried out differential diagnosis of CT data in 107 patients, and also presented epidemiological data from the city statistics department.

\section{Results and Discussions}

For 2020 year, in city Osh, 5511 cases of COVID-19 were registered. Among these cases, 3347 cases were laboratory proven (576 cases independently addressed), imported cases were 35 , self-referral was 1687 , contacts with patients were 1625, 2164 cases based on clinical and epidemiological analyzes. The lethal outcome was 67 (3.09\%) (U 07.1 were 24 cases and U 07.2 were 43 cases).

As shown in Figure 1, during the first wave of COVID-19, the largest peak in the registration of patients in Osh was in July 2020, as throughout Kyrgyzstan, of which patients identified on a clinical and epidemiological basis (U07.2) amounted to 1114 (24.2\%), and COVID positive (U07.1) patients were 845 (15.3\%).

According to CT data, we carried out differential diagnostics in 107 patients, the largest part were patients with only signs of coronavirus pneumonia, in 87 $(81.3 \%)$ cases there was a "frosted glass" symptom. There were $9(8.4 \%)$ patients with covid-19 and with a combination of other comorbidities. The number of patients with other pathology without covid-19 was 6 (5.6\%), and there were 5 (4.7\%) without signs of pathology according to the CT scan, which is presented in Figure 2. 


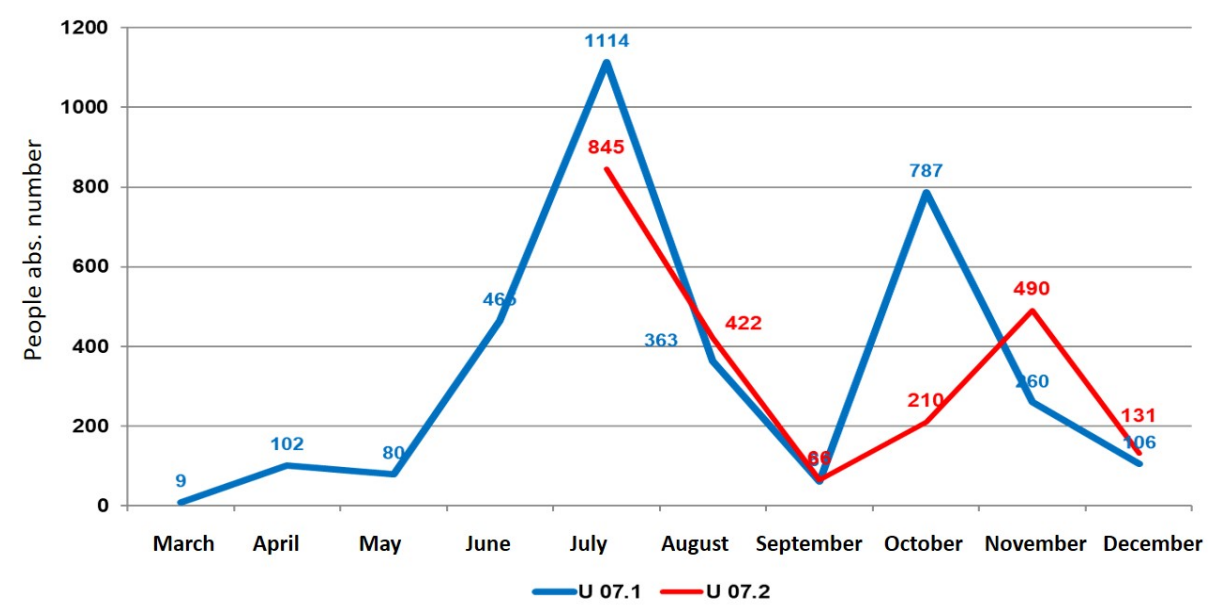

Figure 1. Registration of positive (U 07.1) patients identified on a clinical and epidemiological basis (U 07.2) and COVID by month.

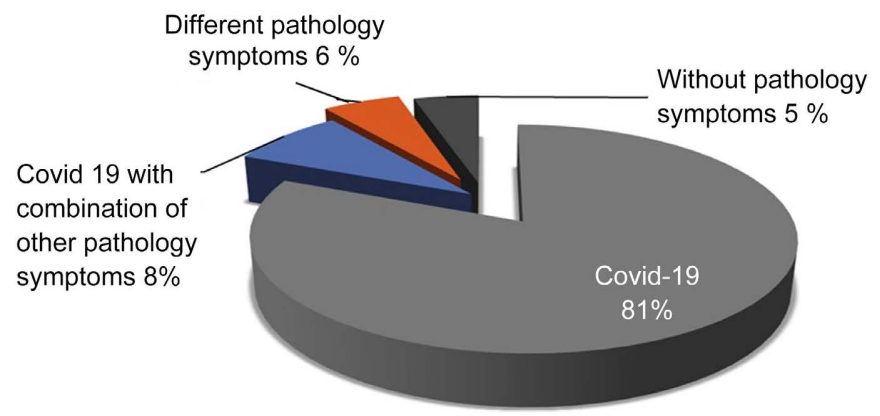

Figure 2. Differential diagnostics results in patients with COVID-19.

Pathognomonic X-ray signs of pneumonia in COVID-19 manifested symptoms: frosted glass, a "cobblestone" pattern, pulmonary vasodilation, traction bronchiectasis, as well as thickened interlobular and intralobular lines in the late stages of disease as a result of pneumosclerosis.

The patients were admitted for 2 weeks in hospital. CT analyses, chest X-ray, $\mathrm{C}$ reactive protein, $\mathrm{D}$ dimer and PCR diagnostics were used for improvement of COVID-19 diagnostics.

\subsection{Symptom "Frosted Glass"}

Symptom "frosted glass" is a slight increase in the density of lung tissue while maintaining the visibility of the walls of blood vessels and bronchi in the area of pathological changes. A ground-glass opacity is the most common (81.3\%) finding in COVID-19 infections (Figures 3(a)-(c). They were usually located bilaterally, multifocally and peripherally, but at an early stage of the disease can manifest as a unifocal lesion, most often localized in the lower lobe of the right lung (43\%). Symptom "frosted glass" most often (52\%) was combined with reticular striation, as in viral interstitial pneumonia.

The picture of "cobblestone pavement" was determined in $48 \%$ of patients, which is indicated in Figure 4(a) (arrows are indicated). 
Sometimes there are thickened interlobular and intralobular lines (Figure 4 (b)) in combination with a frosted glass pattern; this pattern manifests itself as a later stage of disease as manifestations of pneuma fibrosis.

The next CT sign of pneumonia in COVID-19 was the expansion of the pulmonary vessels, which were detected in $48 \%$ of cases. Expansion of the vessel is a typical finding in the area of frosted glass vasodilation (Figure 5(a), Figure 5(b)).

The next CT sign of pneumonia in COVID-19 was traction bronchiectasis, which was identified in $34 \%$ of cases. Traction bronchiectasis is another common finding in the region of the frosted glass area (Figure 6).
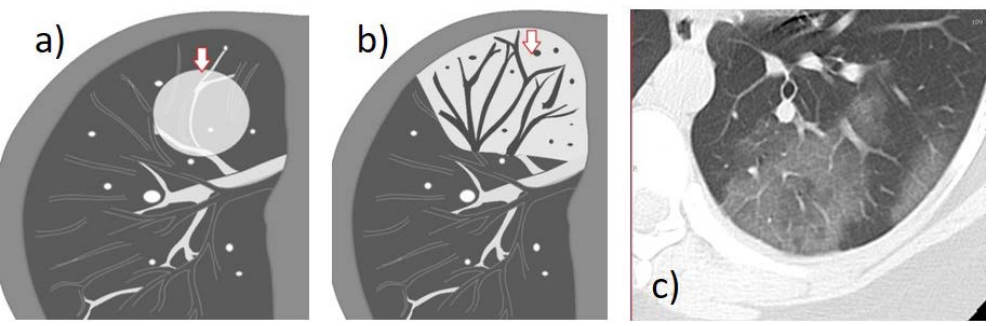

Figure 3. A ground-glass opacity is found in COVID-19 infections.

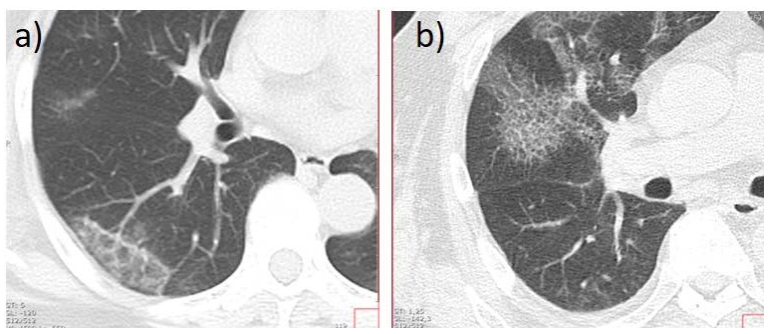

Figure 4. Cobblestone pavement showed in CT image in a patient with COVID-19.

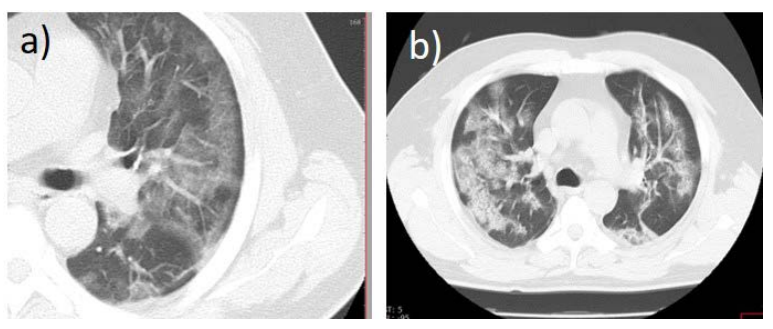

Figure 5. Expansion of the vessel in the area of frosted glass vasodilation a) and b) indicated by arrows.

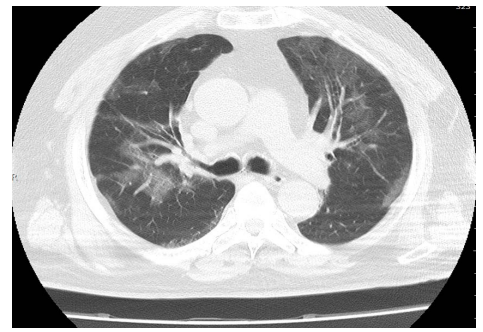

Figure 6. Traction bronchiectasis is another common finding in the region of frosted glass area. 


\section{2. "Frosted Glass" Symptom Differential Diagnosis}

This symptom can occur in many cases, one of which is an incorrect inhalation during CT scanning of a patient, the picture obtained from the patient's exhalation, which can be confused with a "frosted glass" symptom. Incorrect inhalation, i.e. removal of CT during exhalation (Figure 7), "ground glass" can cause hypoaeration during exhalation.

Normally, such a moment can still occur when taking a CT scan in the prone position, which is most often performed for patients with problems of the spinal column, in the postoperative period, and so on. This is called frosted glass functional hypoventilation-gravity (Figure 8):

Also, a frosted glass symptom can be observed in many lung pathologies, such as adenocarcinoma of the lungs, radiation pneumonitis, hypersensitive pneumonitis, cardiogenic pulmonary edema, alveolar hemorrhage and eosinophilic pneumonia, etc.

\subsection{Radiation Pneumonitis in Frosted Glass Symptom}

One of the pathologies represented by "frosted glass" symptom is radiation pneumonitis (Figure 9(a) and Figure 9(b)), which occurs in patients with cancer of the lungs or breast due to radiation and/or chemotherapy, leading to damage to the structure and function of the lung tissue after weeks, months and even years after the treatment. The difference between the "frosted glass" symptom in radiation pneumonitis from the "frosted glass" symptom in COVID-19 pneumonia, firstly (taking into account the history) it is located more medially, often one-sided and has large dimensions.

The next pathology, where the "frosted glass" symptom is also encountered, is cardiogenic pulmonary edema shown in Figure 10(a) and Figure 10(b)). It is manifested by bilateral opacification of ground glass, reflecting extravascular fluid in the alveolar spaces and is located in the lower and posterior parts of the lungs.

These opacities are usually more centrally distributed with peripheral parenchyma and do not correspond to the full mandatory feature of COVID-19, which is the location of the ground glass close to the pleural surfaces. A list of accompanying symptoms associated signs indicating cardiogenic edema:

1) Thickening of the interlobular septum;

2) Diffuse vasodilation;

3) Swollen lymph nodes;

4) Structural cardiac pathology;

5) Fluid in the pleural cavity;

6) The clinical picture of the patient shows the phenomenon of CVS (edema in the legs, and other types of edema, arrhythmia, tachycardia, etc.).

The next pathology is Alveolar hemorrhage manifesting itself with a speckled frosted glass along with the bronchovascular bundles. Figure 11 shows alveolar hemorrhage with spotted frosted glass along with the bronchovascular bundles in a patient with secondary vasculitis and systemic lupus erythematosus. 


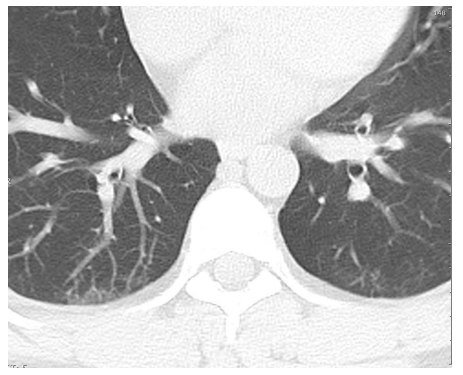

Figure 7. Example of ground glass" which can cause hypoaeration during exhalation.
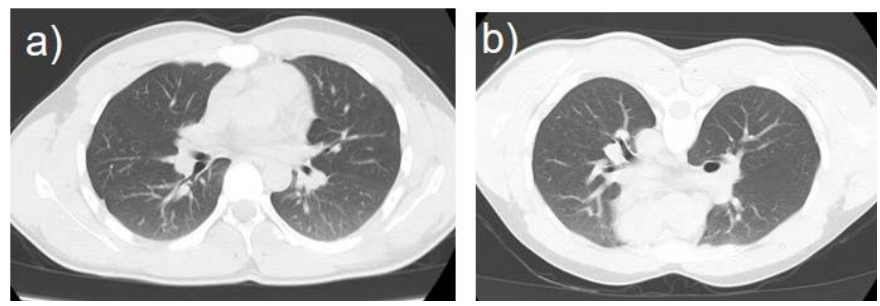

Figure 8. Example of frosted glass functional hypoventilation-gravity (a) on the back; (b) on the stomach.
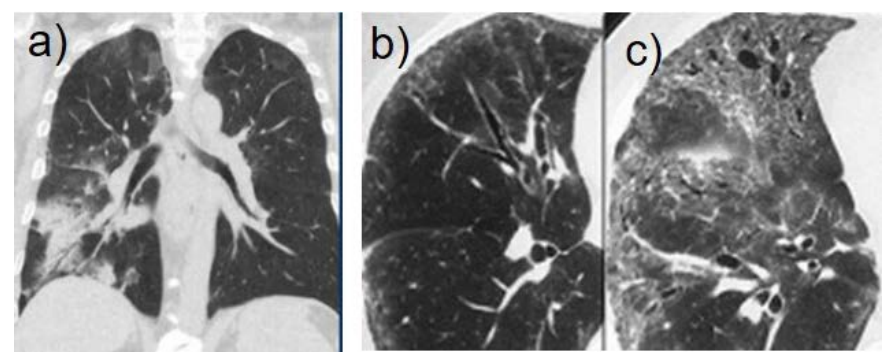

Figure 9. Example of radiation pneumonitis in frosted glass symptom.
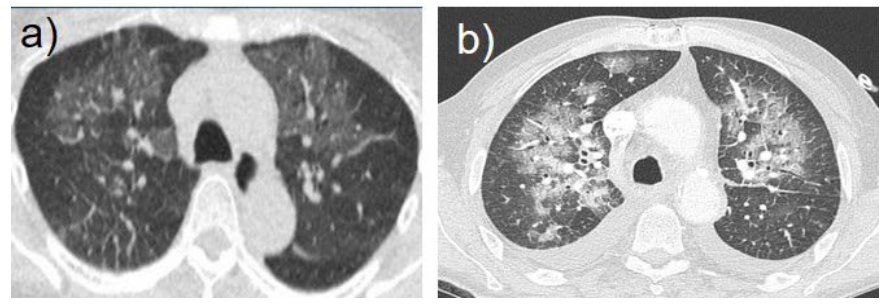

Figure 10. Cardiogenic pulmonary edema.

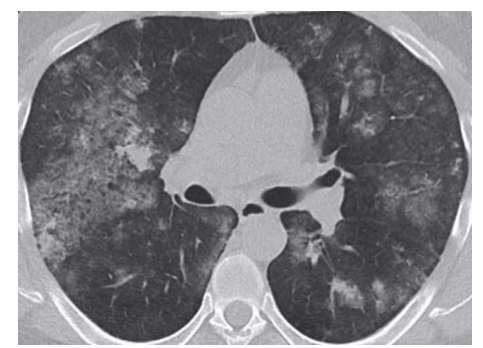

Figure 11. Spotted frosted glass along with the bronchovascular bundles. 


\section{Conclusions}

The presented clinical examples demonstrate difficulty in establishing a true diagnosis in the ground-glass changes in lungs during the COVID-19 pandemic and, as a consequence, the possibility of overdiagnosis. It is also necessary to monitor the dynamics of changes in lung tissue using $\mathrm{CT}$ in patients with COVID-19 for the timely correction of management tactics.

CT data at an early stage of disease development dominated by bilateral, basal peripheral changes of the ground glass type usually are small in area, which is precursor in development of viral pneumonia in COVID-19, as well as multifocal location of ground glass. As the disease progresses, lung tissue areas change like "scraped blanket" type, and areas of consolidation resembling manifestations of organizing pneumonia as "reverse halo" causing diffuse damage of alveoli to develop in 13 to 16 days. Additionally, an example of brain damage is shown in a patient 16 years old with confirmed COVID-19 in the form of acute necrotizing encephalopathy according to magnetic resonance imaging.

\section{Conflicts of Interest}

The authors declare no conflicts of interest regarding the publication of this paper.

\section{References}

[1] Chen, N., Zhou, M., Dong, X., Qu, J., Gong, F., Han, Y., Qiu, Y., Wang, J., Liu, Y., Wei, Y., Xia, J., Yu, T., Zhang, X. and Zhang, L. (2020) Epidemiological and Clinical Characteristics of 99 Cases of 2019 Novel Coronavirus Pneumonia in Wuhan, China: A Descriptive Study. Lancet, 395, 507-513. https://doi.org/10.1016/S0140-6736(20)30211-7

[2] Lotfi, M., Hamblin, M.R., and Rezaei, N. (2020) COVID-19: Transmission, Prevention, and Potential Therapeutic Opportunities. Clinica Chimica Acta, 508, 254-266. https://doi.org/10.1016/j.cca.2020.05.044

[3] Li, H., Liu, S.M., Yu, X.H., Tang, S.L. and Tang, C.K. (2020) Coronavirus Disease 2019 (COVID-19): Current Status and Future Perspectives. International Journal of Antimicrobial Agents, 55, Article No. 105951. https://doi.org/10.1016/j.ijantimicag.2020.105951

[4] Vinokurov, A.S., Belenkaya, O.I., Zolotova, E.A. and Michurina, S.V. (2020) Differential Diagnosis of Bilateral Lung Changes on the Experience of a Hospital Receiving Community-Acquired Pneumonia-not only COVID-19. Medical Imaging, 24, 78-94. https://doi.org/10.24835/1607-0763-2020-2-78-95

[5] Vorotyntseva, N.S. and Goliev, S.S. (2009) Radiopulmonology: Strategy and Tactics for Obtaining and Analyzing an X-Ray Image in Pulmonology. Medical Information Agency, Moscow, 280 p.

[6] Semishcheva, N.L., Karapetyan, E.I., Maltseva, T.A., Avdeeva, N.V. and Odireev, A.N. (2014) Post-Radiation Pneumonitis in the Practice of a Pulmonologist. Far Eastern Scientific Center of Respiratory Physiology and Pathology Bulletin, 53, 137-142.

[7] Kharchenko, V.P. (1998) Radiation Pneumonitis and Pneumofibrosis after Combined Organ-Preserving Treatment of Stage 1 Cancer Patients. Journal of Russian Oncology, 4, 43-47. 
[8] Inciardi, R.M., Lupi, L., Zaccone, G., Italia, L., Raffo, M., Tomasoni, D., Cani, D.S., Cerini, M., Farina, D., Gavazzi, E., Maroldi, R., Adamo, M., Ammirati, E., Sinagra, G., Lombardi, C.M. and Metra, M. (2020) Cardiac Involvement in a Patient with Coronavirus Disease 2019 (COVID-19). JAMA Cardiology, 5, 819-824.

https://doi.org/10.1001/jamacardio.2020.1096

[9] Poyiadji, N., Shahin, G., Noujaim, D., Stone, M., Patel, S. and Griffith, B. (2020) COVID-19-Associated Acute Hemorrhagic Necrotizing Encephalopathy: CT and MRI Features. Radiology, 296, E119-E120.

https://doi.org/10.1148/radiol.2020201187

[10] Quispe-Cholan, A., Anticona-De-La-Cruz, Y., Cornejo-Cruz, M., Quispe-Chirinos, O., Moreno-Lazaro, V. and Chavez-Cruzado, E. (2020) Tomographic Findings in Patients with COVID-19 According to Evolution of the Disease. The Egyptian Journal of Radiology and Nuclear Medicine, 51, Article No. 215. https://doi.org/10.1186/s43055-020-00329-5

[11] Atakla, H.G., Condé, K., Noudohounsi, M., Dongmo, M., Garba, A.H., Houinato, D.S. and Cissé, F.A. (2020) Interest of the Thoracic Scanner in the Diagnosis of COVID-19: Study of 35 Cases in the Republic of Guinea. The Pan African Medical Journal, 35, Article No. 133. https://doi.org/10.11604/pamj.supp.2020.35.2.24549 